Nouvelles perspectives en sciences sociales

Revue internationale de systémique complexe et d'études relationnelles

\title{
Aide à la décision multicritère : cohérence et équité grâce à l'analyse de concepts
}

\section{Mireille Ducassé et Sébastien Ferré}

Volume 5, numéro 2, mai 2010

Sur le thème de la simulation

URI : https://id.erudit.org/iderudit/044082ar

DOI : https://doi.org/10.7202/044082ar

Aller au sommaire du numéro

Éditeur(s)

Prise de parole

ISSN

1712-8307 (imprimé)

1918-7475 (numérique)

Découvrir la revue

Citer cet article

Ducassé, M. \& Ferré, S. (2010). Aide à la décision multicritère : cohérence et équité grâce à l'analyse de concepts. Nouvelles perspectives en sciences sociales, 5(2), 181-196. https://doi.org/10.7202/044082ar

\section{Résumé de l'article}

De nombreuses décisions sont prises en commission, par exemple pour affecter des ressources. Les critères de décision sont difficiles à exprimer et la situation globale est en général trop complexe pour que les participants puissent l'appréhender pleinement. Dans cet article, nous décrivons un processus de décision pour la sélection de candidats à un emploi. L'analyse de concepts y est utilisée pour faire face aux problèmes mentionnés ci-dessus. Grâce à l'analyse formelle de concepts et aux systèmes d'information logiques, les personnes fair play ont la possibilité d'être équitables envers les candidats et de faire preuve de cohérence dans leurs jugements sur toute la durée du processus de décision. 


\section{Aide à la décision multicritère : cohérence et équité grâce à l'analyse de concepts}

Mireille Ducassé

Institut national des sciences appliquées, Rennes

Sébastien FerRé

Université de Rennes 1

T existe de nombreuses situations où les décisions sont prises

en commission, par exemple, pour embaucher des personnes ou affecter des ressources. Ces décisions font généralement intervenir de multiples critères non nécessairement comparables entre eux. Les décisions finales de ces commissions ont nécessairement une part d'arbitraire. Alors que certaines personnes peuvent se réjouir d'avoir l'occasion de développer des stratégies de manipulation, notre expérience est que la plupart des participants essayent d'être aussi honnêtes que possible afin de prendre une bonne décision pour la collectivité. Cet article est dédié à ces participants fair play qui sortent souvent des réunions en étant frustrés. Condorcet et Arrow, pour ne citer qu'eux, ont démontré que, quelle que soit la méthode utilisée, il y a toujours certains critères rationnels qui ne sont pas satisfaits. Cela explique en partie ces frustrations. Nous conjecturons donc que ce qui tracasse les participants n'est pas tant de ne pas avoir trouvé la meilleure solution que les incohérences et les iniquités qui peuvent avoir eu lieu. 
Les critères de décision sont difficiles à exprimer. De plus, la situation est, en général, trop complexe pour que les participants puissent l'appréhender dans sa globalité et la garder en tête à tout moment de la discussion. Les méthodes de la théorie du choix social en recherche opérationnelle ${ }^{1} s^{\prime}$ attaquent à ce problème par une modélisation numérique. Pour chaque critère, une préférence exprimée sous forme de nombre réel est déterminée. Un traitement numérique permet d'attribuer un score à chaque choix possible. Comme le soulignent Bana e Costa et Chagas ${ }^{2}$, ces nombres ont un côté artificiel. Ces auteurs proposent une méthode, appelée MACBETH, qui permet aux personnes concernées par le choix de poser des jugements non numériques. Le logiciel transforme ensuite ces jugements en nombres, vérifiant au passage un certain nombre de propriétés comme, par exemple, l'absence de conflit. Malgré ses avantages, cette approche ne nous semble pas la mieux adaptée au fonctionnement en commission. Une commission est en général mise en place quand il n’y a pas de meilleure solution évidente. Le rôle de la commission est donc, bien entendu, de prendre une décision, mais surtout d'en endosser collectivement la responsabilité. Une fois les nombres produits, les raisons et les argumentations qui ont amené à produire ces nombres peuvent être perdues. De plus, les calculs utilisant les préférences et les poids relatifs des critères peuvent donner des résultats obscurs et difficiles à défendre.

Dans cet article, nous décrivons un processus de décision où

$1 \quad$ Voir par exemple Bernard Roy et Denis Bouyssou, Aide multicritère à la décision : méthodes et cas, Paris, Economica, 1993; Michel Grabisch, "Une approche constructive de la décision multicritère ", Traitement du Signal, vol. 22, n 4, 2005, p. 321-337 ou Denis Bouyssou, Thierry Marchant et Patrice Perny, «Aide multicritère à la décision et théorie du choix social », dans Denis Bouyssou, Didier Dubois, Marc Pirlot et Henri Prades (dir.), Concepts et méthodes pour l'aide à la décision, IC2 Séries, n 5, Paris, Hermès, 2006.

2 Carlos A. Bana e Costa et Manuel P. Chagas, "A Career Choice Problem: An Example of How to Use MACBETH to Build a Quantitative Value Model Based on Qualitative Value Judgments ", European Journal of Operational Research, vol. 153, n 2, 2004, p. 323-331. 
l'analyse logique de concepts ${ }^{3}$ et l'analyse formelle de concepts ${ }^{4}$ sont utilisées pour que les résultats puissent être cohérents et équitables, sans avoir besoin de modéliser a priori les préférences individuelles. La commission travaille sur un ensemble de critères qui sont renseignés par des faits. Ces faits sont traités de manière symbolique. Ils ont leur type "naturel ». Seuls sont numériques ceux qui le sont intrinsèquement, par exemple, le nombre de publications dans une revue. Cela est rendu possible par les fondements théoriques de l'analyse logique de concepts qui utilise la logique pour représenter la description des objets. De plus, la logique donne des bases solides pour permettre de poser des requêtes et de créer des liens de navigation, ainsi que pour raisonner.

Dans la suite, nous illustrons notre approche par un exemple qui reconstitue une réunion de commission de recrutement pour un poste d'attaché temporaire d'enseignement et de recherche. Pouvaient candidater, soit des docteurs, soit des doctorants sur le point de soutenir. Il y a eu 43 candidats. Au département informatique de l'Institut national des sciences appliquées de Rennes, outre un rapport écrit, les rapporteurs remplissent aussi une feuille de calcul de tableur où un certain nombre de critères objectifs sont évalués. Lors de la réunion d'origine, le seul outil utilisé a été un tableur. Les arguments exprimés au cours des discussions ont été reconstitués a posteriori. Une version plus détaillée de cette étude de cas existe.

Deux outils sont utilisés, Camelis et Conexp. Le premier, Camelis ${ }^{6}$, s'appuie sur l'analyse logique de concepts pour faciliter

3 Sébastien Ferré et Olivier Ridoux, «An Introduction to Logical Information Systems ", Information Processing \& Management, vol. 40, $\mathrm{n}^{\circ}$ 3, 2004, p. 383-419.

4 Bernhard Ganter et Rudolf Wille, Formal Concept Analysis: Mathematical Foundations, Heidelberg, Springer, 1999.

5 Mireille Ducassé et Sébastien Ferré, « Fair(er) and (Almost) Serene Committee Meetings with Logical and Formal Concept Analysis ", International Conference on Conceptual Structures, Springer-Verlag, Lecture Notes in Artificial Intelligence $\mathrm{n}^{\circ}$ 5113, juillet 2008.

6 Une version gratuite peut être téléchargée depuis http://www.irisa.fr/LIS/ ferre/camelis. Sébastien Ferré, « Camelis: A Logical Information System to Organize and Browse a Collection of Documents ", International Journal of 
l'organisation d'une collection d'objets ainsi que la navigation dans cette collection. Le second, Conexp ${ }^{7}$, développé par Sergey A. Yevtushenko, permet, entre autres, d'éditer un contexte formel et d'afficher des treillis de concepts.

\section{Processus de décision préconisé}

Le processus de décision que nous préconisons a trois étapes : d'abord, une analyse axée sur les attributs (ou les critères) qui élimine les candidats manifestement hors profil, ensuite une analyse axée sur les candidats, enfin, une discussion pour classer les candidats restants.

Il n'est pas possible d'examiner tous les attributs de chaque candidat de manière détaillée. Cela nécessiterait au moins 5 minutes par candidat. Avec plus de 40 candidats, cela voudrait dire plusieurs heures d'analyse. Passer autant de temps sur les candidats manifestement hors profil serait improductif. Partir d'une analyse axée sur les attributs contribue à accélérer le processus de manière équitable et à passer du temps sur les candidats dans le profil.

\subsection{Analyse axée sur les attributs}

Dans la première étape, l'analyse est axée sur les attributs. Les attributs sont examinés tour à tour. Pour chacun d'eux la commission décide de la pertinence de l'attribut pour la décision à prendre. En particulier, il est décidé si un attribut est :

- sélectif : la commission décide que c'est un critère obligatoire pour ce concours. Les candidats qui ne le remplissent pas sont éliminés.

- sélectif mais compensé : la commission décide que, dans l'absolu, l'attribut devrait être obligatoire, mais, dans ce contexte d'autres attributs présentés par certains candidats pourraient compenser l'absence de cet attribut. Les attributs compensatoires sont spécifiés. Les candidats qui ne

7 Une version gratuite peut être téléchargée depuis http://conexp.sourceforge. net/. 
remplissent ni l'attribut sélectif, ni l'un des attributs compensatoires sont éliminés.

- pertinent : la commission décide que l'attribut est pertinent mais pas obligatoire. Il est conservé pour différencier les candidats dans la suite des discussions.

- non pertinent : la commission décide que l'attribut n'est pas pertinent pour cette décision.

En outre, de nouveaux attributs peuvent être identifiés et le tableau conséquemment mis à jour à la volée.

Figure 1

\section{Aperçu de Camelis pendant l'analyse axée sur les attributs}

La fenêtre du haut est la zone de requête, la fenêtre en bas à droite est la zone des

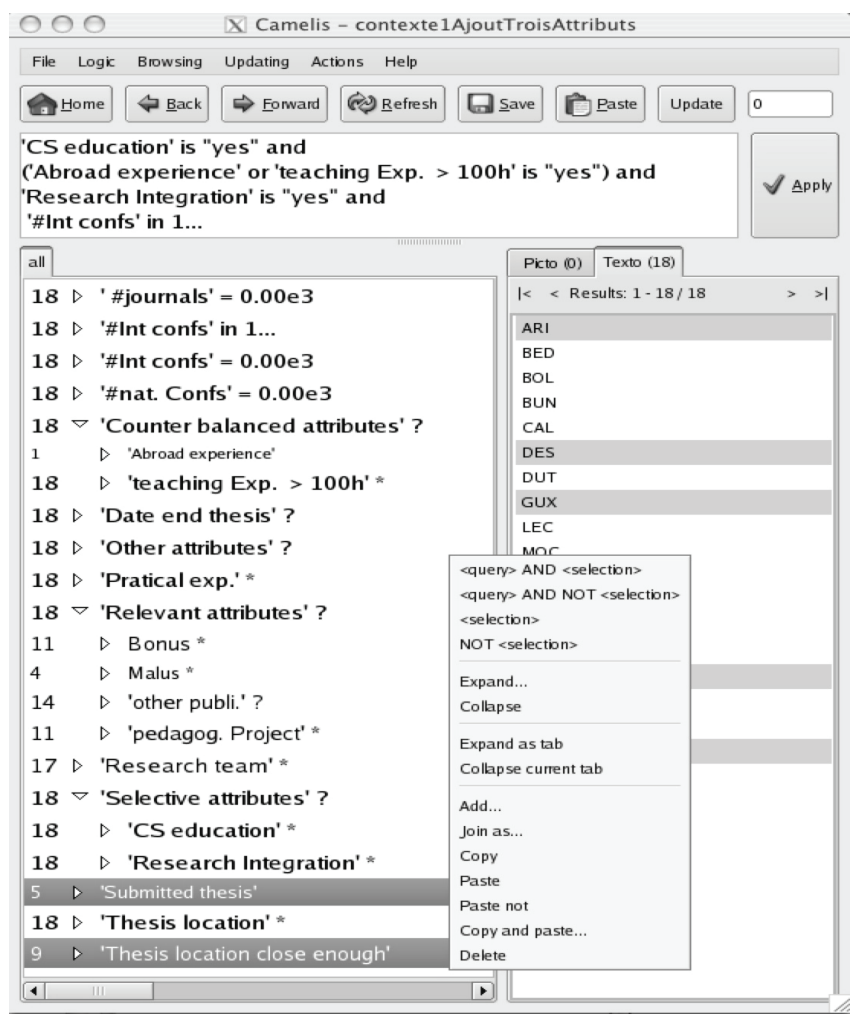

candidats, la fenêtre en bas à gauche contient la taxonomie de tous les attributs, la fenêtre pop up contient les commandes accessibles lorsque des attributs sont sélectionnés. 
Dans notre exemple, cette première étape se fait sous Camelis. La figure 1 montre un instantané pris au cours de cette étape axée sur les attributs. La fenêtre du haut est la zone de requête, elle contient les critères de sélection que la commission a déjà spécifiés à cet instant. Sur la figure, cela peut être lu comme "les candidats sélectionnés doivent avoir une formation initiale en informatique ET (ils doivent avoir une expérience à l'étranger OU une expérience d'enseignement) ET leur intégration dans le laboratoire de recherche doit être indiscutable ET ils doivent avoir au moins une publication dans une conférence internationale ». Il est important de noter que cette requête a été construite uniquement par navigation, c'est-à-dire en cliquant sur des éléments de la zone des attributs (voir plus bas).

La fenêtre en bas à droite est la zone des candidats. Elle contient le nom codé des candidats qui remplissent les critères de la zone de requête. On peut voir que, sur les 43 candidats initiaux, seulement 18 satisfont la requête ci-dessus.

La fenêtre en bas à gauche contient la taxonomie de tous les attributs. Le nombre à gauche de chaque attribut indique combien de candidats encore en lice possèdent l'attribut. On peut voir, par exemple, que seulement 11 candidats, des 18 restants, ont un bonus. La figure illustre, entre autres, la façon dont deux nouveaux attributs seront pris en compte dans la requête. La commission a tenu à ne sélectionner que des candidats ayant déjà soumis leur mémoire ou travaillant sur leur thèse à proximité de Rennes, pour leur laisser de meilleures chances de terminer dans de bonnes conditions. Deux nouveaux attributs, Submitted thesis et Thesis location close enough ont été introduits en cours de réunion. Sur la figure, les deux attributs ont été cliqués. Camelis les a grisés. Il a également grisé les candidats ne remplissant pas l'une ou l'autre des propriétés. La commission peut donc voir qui sera éliminé si la disjonction des deux attributs est jugée sélective. "ARI ", " DES ", "GUX » et deux autres candidats dont le nom est caché par la fenêtre $p o p-u p$ risquent de disparâ̂tre. Chaque rapporteur a une chance de signaler si un candidat qu'il estime valable sera perdu. Un clic-droit ouvre la fenêtre $p o p$ 
up. L'utilisateur peut cliquer sur "<query> AND < selection> ", ce qui ajoutera "AND (Submitted thesis OR Thesis location close enough) " à la requête.

\subsection{Analyse axée sur les candidats}

À un certain point, le nombre de candidats restants est assez petit pour qu'il devienne faisable d'examiner les candidats de manière détaillée. La commission analyse alors tous les attributs de chaque candidat, tour à tour. Ce faisant, la commission peut, bien sûr, encore décider qu'un attribut doit être sélectif ou sélectif mais compensé. Au cours de la première phase, axée sur les attributs, la commission vérifie que les candidats qui sont sur le point d'être éliminés n’ont effectivement pas l'attribut sélectif requis. Au cours de la deuxième phase, axée sur les candidats, la commission vérifie que les candidats restants ont effectivement les attributs que leurs rapporteurs leur ont attribués. La commission vérifie également qu'il ne manque pas d'association candidat-attribut importante. Il est fort probable que de nouveaux attributs émergent.

Nous illustrons la deuxième étape, axée sur les candidats, par deux événements intéressants lors de la réunion d'origine. Lors de l'examen de chaque candidat, la commission a jugé que les rapporteurs avaient été un peu trop optimistes en ce qui concernait le potentiel d'intégration dans le laboratoire de recherche de deux candidats. Les deux candidats considérés ont donc perdu cet attribut en séance. Par ailleurs, quelqu'un parmi la commission a fait remarquer que l'un des candidats restants avait une " contribution majeure à l'enseignement ». La commission a approuvé et considéré qu'il serait intéressant de conserver cette information. Un attribut a été ajouté. Il faut souligner qu'il a été ensuite attribué à tous les candidats ayant une contribution importante à l'enseignement.

1.3. Classement : transformation des ordres partiels en ordre total

À un certain point, la commission est convaincue que les informations sur lesquelles elle travaille sont valides et que la requête 
de sélection est pertinente pour la décision à prendre. En outre, il ne peut plus être fait de sélection objective avec un consensus général. Nous conjecturons que, à ce stade, le treillis de concepts formels peut être utile car il donne une vue d'ensemble de tous les ordres partiels et il est devenu suffisamment petit pour être lisible. La tâche de la commission est de classer cinq candidats; il s'agit, par conséquent, de transformer les ordres partiels en un ordre total.

Figure 2

Treillis de concepts avec les quatre derniers candidats

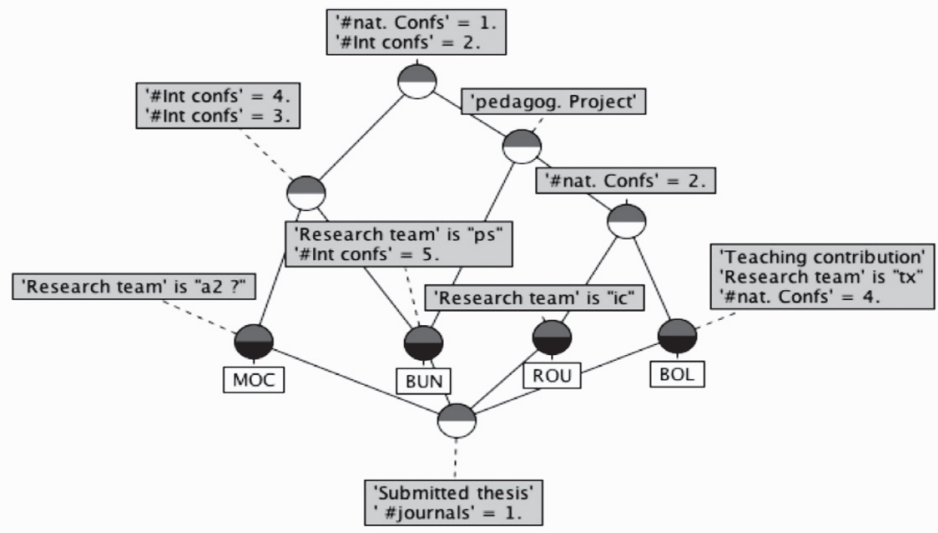

Chaque nœud est un concept formel, tous les candidats atteignables en descendant dans le treillis lui sont associés, tous les attributs atteignables en remontant dans le treillis lui sont associés.

La figure 2 montre le treillis relatif aux quatre derniers candidats ( $\mathrm{MOC}$ », " $\mathrm{BUN}$ », " $\mathrm{ROU}$ » et « $\mathrm{BOL}$ ») avec les attributs qui ont semblé les plus pertinents à la commission. Le treillis se lit comme suit. Chaque noud est un concept formel; tous les candidats atteignables en descendant dans le treillis lui sont associés; tous les attributs atteignables en remontant dans le treillis lui sont associés. Pour un candidat donné, ses attributs se trouvent donc en remontant dans le treillis. Par exemple, les attributs de "BOL " sont "Teaching contribution ", "Research team is "tx" ", "\#nat Confs = 4 ", "\#nat Confs = 2 ", "pedagog. Project ", "\#Int confs = 2 " et "\#nat Confs = 1 ". Cela peut se 
traduire par « le candidat identifié par BOL a une contribution significative en enseignement, il devrait pouvoir s'intégrer dans l'équipe tx, il a publié dans 4 congrès nationaux et deux congrès nationaux, il a un projet pédagogique ». Pour un attribut donné, les candidats qui le possèdent se trouvent en descendant dans le treillis. Par exemple, les candidats qui ont un projet pédagogique, "pedagog. Project", sont " BUN », " ROU » et " BOL ». Le nœud tout en haut du treillis montre les attributs communs à tous les candidats. Le nœud tout en bas du treillis montre les attributs qu'aucun des candidats n'a.

Figure 3

Treillis de concepts avec les deux derniers candidats

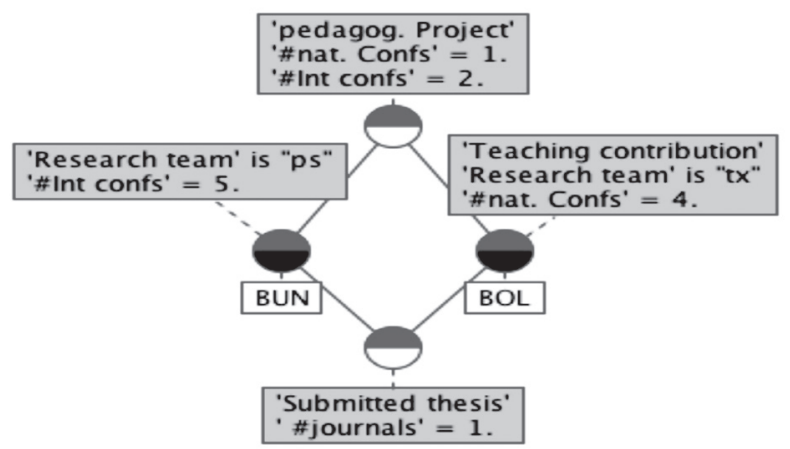

Le nœud tout en haut du treillis montre les attributs communs à tous les candidats, le nœud tout en bas du treillis montre les attributs qu'aucun des candidats n'a, les attributs spécifiques sont attachés à chaque candidat.

$\mathrm{Vu}$ le nombre de publications internationales, la commission a estimé que " $\mathrm{MOC}$ » et " $\mathrm{BUN}$ » étaient meilleurs en matière de recherche. Elle a également estimé que le point fort de " $\mathrm{ROU}$ » et " $\mathrm{BOL}$ " était plutôt l'enseignement à cause du projet pédagogique. En outre, il a été décidé que «BUN » était meilleur que "MOC " compte tenu du nombre de ses publications et de leur qualité. La qualité des publications a été discutée en séance, mais n'est pas reportée sur le treillis. Il a également été décidé que " BOL » était meilleur que « ROU » compte tenu de la contribution à l'enseignement. La commission a donc décidé 
de ne garder que " $\mathrm{BUN}$ » et " $\mathrm{BOL}$ ». Il en est résulté le treillis de la figure 3. Le noud tout en haut indique que les deux candidats ont un projet pédagogique, au moins une publication dans un congrès national et au moins deux publications dans des congrès internationaux. Le nœud tout en bas indique qu'aucun des deux n'a encore soumis son manuscrit de thèse et qu'aucun des deux n'a encore de publication dans une revue. Les attributs spécifiques sont attachés à chaque candidat. À ce stade, la commission a procédé au vote.

\section{Discussion}

Dans cette section, nous discutons des avantages de notre méthode pour prendre des décisions lorsqu'elle est appliquée par des personnes sincères.

\subsection{Le processus est équitable pour les candidats}

Ce processus est équitable pour les candidats. Jusqu'au dernier stade, aucun candidat ne peut être éliminé sans raison explicite et cette raison est appliquée à tous les candidats. Les critères de sélection sont spécifiés explicitement. Tous les candidats remplissant un critère sont traités de manière identique. Lorsque la commission estime qu'une qualité pourrait compenser un critère requis, tous les candidats présentant cette qualité seront considérés de la même manière. Les rapports des candidats éliminés par la sélection courante sont également mis à jour. Si la commission a des doutes et assouplit certains des attributs sélectifs, certains candidats peuvent ne plus être éliminés par les attributs relaxés. Ils peuvent donc réapparaitre dans la sélection. Comme leurs attributs ont été mis à jour, toutes les décisions qui ont été prises après leur élimination leur seront appliquées automatiquement.

2.2. Le processus est cohérent, flexible, réversible, transparent et traçable

Le treillis de concepts sous-jacent assure que le processus est cohérent tout au long de la réunion et que le résultat, en général un ordre total, est compatible avec les ordres partiels, faciles à 
exprimer. La flexibilité et la cohérence sont à la base de l'équité discutée ci-dessus. Par exemple, avec notre approche, un candidat ne peut être éliminé s'il a un attribut qui a permis à un autre candidat d'être repêché. En outre, sans le support d'outil, il est facile de commettre des incohérences en toute innocence au cours de la réunion. Avec notre approche, si un critère est dit essentiel au début de la discussion et si les candidats sélectionnés ne le satisfont pas, à tout le moins, cela sera visible et la commission pourra en discuter. De plus, le résultat de la sélection est indépendant de l'ordre dans lequel les décisions atomiques ont été prises. En conséquence, toutes les décisions partielles peuvent être remises en question à tout moment, le processus est réversible. Il n'est pas nécessaire d'être toujours sur le qui-vive; il n'y a pas de décision fatale jusqu'à la dernière minute. Certaines réunions de commission ressemblent parfois à un jeu de bonneteau. Notre processus est transparent et traçable. Le contexte peut être facilement étendu à la volée avec de nouveaux attributs et de nouvelles associations entre attributs et candidats. Chaque membre de la commission peut suivre les mises à jour et peut soulever une objection à tout moment. Le nouveau contexte est facile à vérifier. Les décisions de sélection sont visibles dans la zone de requête qui exprime la logique de la décision en cours.

2.3. Le résultat final provient de décisions nombreuses, petites et (relativement) faciles

N'importe qui peut proposer un nouvel attribut ou une nouvelle association, ou suggérer qu'un attribut doit être sélectif. Si la commission accepte par consensus, la proposition est validée. Si aucun consensus ne se dégage, la commission peut voter pour décider si un nouvel attribut est pertinent ou sélectif. Elle peut aussi voter pour décider si un candidat a bien un attribut. Ce sont de petites décisions, relativement faciles à prendre. En outre, si la commission décide qu'un attribut n'est pas sélectif, mais seulement pertinent, il est raisonnable que cette information apparaisse explicitement. Elle sera prise en compte plus tard lors du vote. Il est aussi pertinent qu'un critère soit étiqueté pertinent 
même si une seule personne dans la commission juge qu'il en est ainsi. Le consensus est seulement obligatoire pour les critères sélectifs. Chaque membre de la commission utilisera l'information contenue dans les attributs pertinents comme il l'entend au moment du vote. Cela permet d'économiser beaucoup de vaines discussions.

\subsection{Les outils d'analyse de concepts sont pertinents}

L'ensemble du processus est très difficile à réaliser sans outils appropriés. Utiliser un tableur a été déjà une grande amélioration sur les rapports informels oraux ou écrits. Toutefois, lors des manipulations dans le tableur, il est très facile de faire des erreurs et de ne pas s'en rendre compte. De ce fait, il est difficile d'être sûr que le processus reste cohérent et correct. En outre, ajouter des attributs et des associations dans le tableau est très fastidieux et, de nouveau, sujet à erreurs. Pire encore, le suivi du processus de sélection est presque impossible, surtout quand la sélection fait intervenir une combinaison des connecteurs logiques ET, OU et NON. Dans Camelis, tout ce qui a été si difficile à faire avec un tableur se fait aisément. En outre, la cohérence globale est garantie. Enfin, la requête conserve l'historique des décisions et permet ainsi de donner une explication de ce qui s'est passé, au moins jusqu'au vote. L'utilisation des outils nécessite de remplir un tableau qui renseigne les critères factuels. Cela peut s'avérer coûteux. Il est, toutefois, relativement courant de fabriquer des tableaux comparatifs lorsqu'un choix doit être fait, comme le montrent abondamment les magazines de défense des consommateurs. De plus, Camelis peut être utilisé directement pour saisir les rapports.

2.5. Utiliser des outils entièrement automatiques ne serait pas adapté à ce type de commission

Il est important de noter que, même avec une grande expérience des commissions, il est hors de question que le tableau initial puisse être rempli sans être examiné collectivement. Les informations doivent être mises à jour et validées. Tout d'abord, il est 
impossible de garantir que tous les critères importants avaient été prévus et anticipés avant que les rapports ne soient faits. Deuxièmement, la situation dépend de l'ensemble des candidats : il est possible qu'un nombre insuffisant de candidats remplisse les critères de sélection qui ont été utilisés lors de réunions précédentes. Troisièmement, certains des candidats peuvent présenter des caractéristiques intéressantes qui n'avaient pas été identifiées auparavant. Quatrièmement, les rapporteurs peuvent faire des erreurs, en toute innocence, en remplissant le tableau. En conséquence, il est hors de question que la décision soit prise automatiquement à partir du tableau tel qu'il est en début de réunion. L'un des objectifs du processus décrit est, justement, que les membres de la commission se mettent collectivement d'accord à la fois sur un ensemble d'attributs/de critères et sur qui les satisfait. Le tableau révisé au cours du processus est un des résultats de la réunion, presque aussi important que la liste classée des candidats retenus. Une fois que le tableau est accepté par la commission, tous les attributs sont identifiés et tous les attributs sélectifs ont été acceptés. On peut penser qu'à ce stade chaque membre de la commission s'est fait une opinion sur le poids respectif des attributs qui ont été étiquetés comme pertinents. Les outils de recherche opérationnelle peuvent donc être utilisés, car les préférences pour chaque critère, si elles étaient exprimées, formeraient bien un préordre complet. Toutefois, pour ce type de commission, il ne nous semble pas pertinent d'utiliser une méthode qui calcule des scores globaux. En effet, la commission devrait encore se mettre d'accord sur un poids pour chaque critère. Le coût et la durée d'une telle négociation ne semblent pas acceptables pour le recrutement d'un attaché temporaire d'enseignement et de recherche (ATER). Cela pourrait se discuter dans un autre contexte, par exemple, pour prendre des décisions lourdes comme le tracé d'une ligne de métro. Pour notre cas, à ce stade, le vote semble un compromis acceptable. 


\section{Conclusion}

L'analyse de concepts a été appliquée à de nombreux contextes sociaux, par exemple, aux réseaux sociaux ${ }^{8}$ ou à la communication par le biais d'ordinateur ?'. La plupart de ces applications sont destinées à être appliquées a posteriori, afin d'obtenir une certaine compréhension des phénomènes sociaux étudiés. Au contraire, nous proposons d'utiliser l'analyse logique de concepts et l'analyse formelle de concepts en cours, et comme soutien, des phénomènes eux-mêmes.

Une raison pour ne pas montrer le treillis de concepts global est qu'il est trop grand pour être géré à la main. La figure 4 montre le treillis de concepts représentant les 43 candidats et seulement 40 des 62 attributs; le nombre de concepts, donc de nœuds, est déjà de 1 239. Des vues locales telles que celles proposées par Camelis ou d'autres outils basés sur l'analyse de concepts formels dans le domaine de la recherche d'information $^{10}$ sont mieux adaptés pour les deux premières étapes décrites dans cet article que les treillis globaux.

Il existe de nombreuses situations similaires à celle reconstituée dans cet article. Notre approche pourrait, par exemple, être utilisée chaque fois qu'une ressource rare doit être attribuée par un groupe, s'il est prêt à établir des rapports factuels selon un ensemble de critères même imparfaitement spécifiés avant la réunion de la commission. Nous avons argumenté, qu'avec notre approche, des personnes fair play ont la possibilité d'exprimer les raisons d'une décision. La commission peut être cohérente dans ses jugements au cours de l'ensemble de la réunion. Elle peut être équitable avec les candidats. Ses membres peuvent endosser collectivement la responsabilité de la décision finale.

8 Camille Roth, "Binding Social and Semantic Networks ", European Conference on Complex Systems, septembre 2006.

9 Noriko Hara, "Analysis of Computer-Mediated Communication: Using Formal Concept Analysis as a Visualizing Methodology ", Journal of Educational Computing Research, vol. 26, n ${ }^{\circ}$ 1, 2002, p. 25-49.

10 Jon Ducrou, Björn Vormbrock, et Peter W. Eklund, « FCA-Based Browsing and Searching of a Collection of Images ", International Conference on Conceptual Structures, Springer-Verlag, Lecture Notes in Computer Science $\mathrm{n}^{\circ}$ 4068, 2006. 
Figure 4

Treillis de concepts pour les 43 candidats

et $\mathbf{4 0}$ des 62 attributs
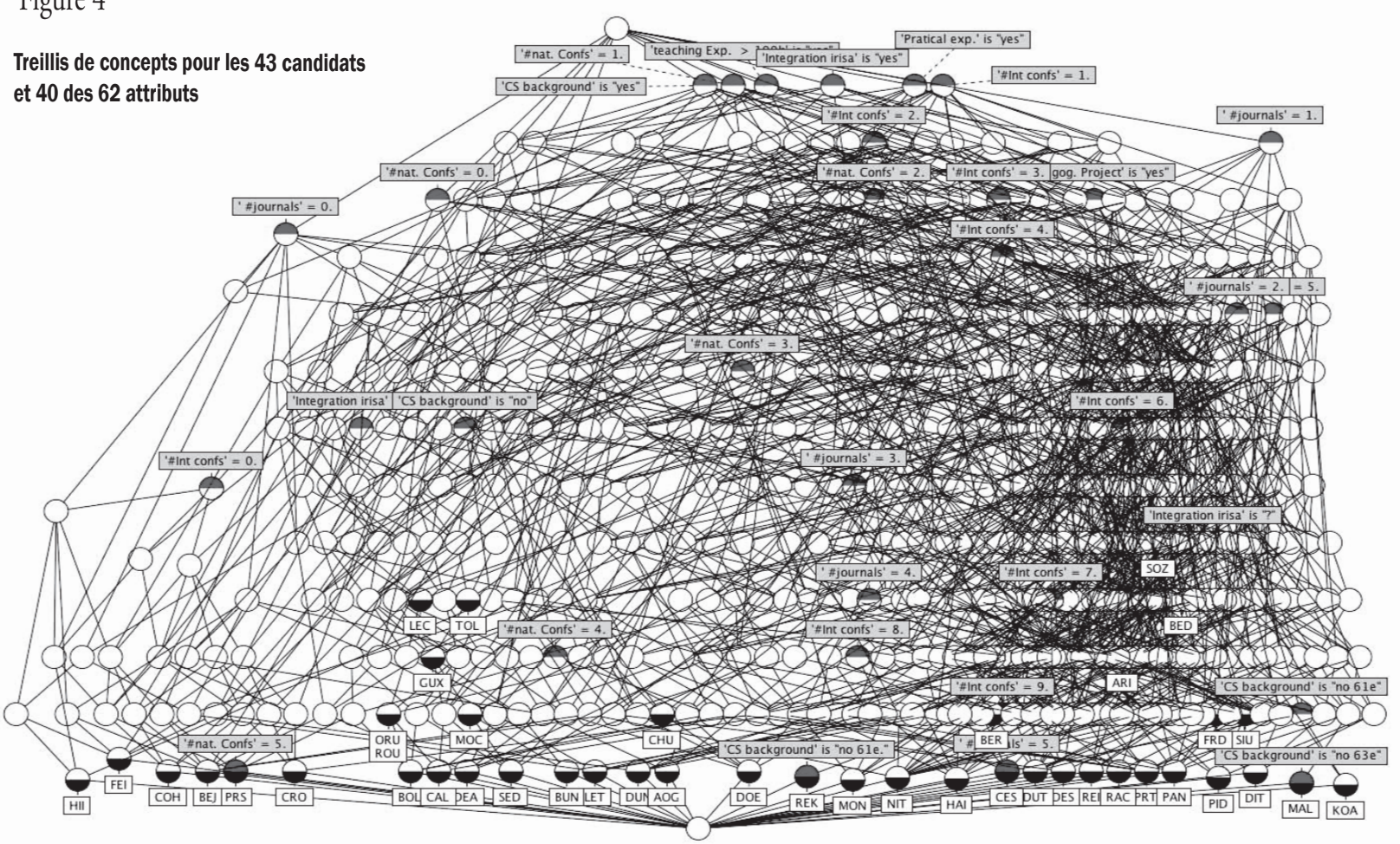


\section{Bibliographie}

Bana e Costa, Carlos A. et Manuel P. Chagas, «A Career Choice Problem: An Example of How to Use MACBETH to Build a Quantitative Value Model Based on Qualitative Value Judgments ", European Journal of Operational Research, vol. 153, no 2, 2004, p. 323-331.

Bouyssou, Denis, Thierry Marchant et Patrice Perny, «Aide multicritère à la décision et théorie du choix social ", dans Denis Bouyssou, Didier Dubois, Marc Pirlot et Henri Prades (dir.), Concepts et méthodes pour l'aide à la décision, IC2 Séries, no 5, Paris, Hermès, 2006.

Ducassé, Mireille et Sébastien Ferré, "Fair(er) and (Almost) Serene Committee Meetings with Logical and Formal Concept Analysis ", International Conference on Conceptual Structures, Springer-Verlag, Lecture Notes in Artificial Intelligence, ${ }^{\circ}$ 5113, 2008.

Ducrou, Jon, Björn Vormbrock et Peter W. Eklund, «FCA-Based Browsing and Searching of a Collection of Images ", International Conference on Conceptual Structures, Springer-Verlag, Lecture Notes in Computer Science, $n^{\circ} 4068$, juillet 2006.

Ferré, Sébastien, «Camelis: A Logical Information System to Organize and Browse a Collection of Documents ", International Journal of General Systems, vol. 38, no 4, 2009, p. 379-403.

Ferré, Sébastien et Olivier Ridoux, «An Introduction to Logical Information

Systems ", Information Processing \& Management, vol. 40, n 3, 2004, p. 383-419.

Ganter, Bernhard et Rudolf Wille, Formal Concept Analysis: Mathematical Foundations, Heidelberg, Springer, 1999.

Grabisch, Michel, "Une approche constructive de la décision multicritère ", Traitement du Signal, vol. 22, no 4, 2005, p. 321-337.

Hara, Noriko, "Analysis of Computer-Mediated Communication: Using Formal Concept Analysis as a Visualizing Methodology ", Journal of Educational Computing Research, vol. 26, n 1, 2002, p. 25-49.

Roth, Camille, "Binding Social and Semantic Networks ", European Conference on Complex Systems, septembre 2006.

Roy, Bernard et Denis Bouyssou, Aide multicritère à la décision : méthodes et cas, Paris, Economica, 1993. 